\title{
SERS+MEF of the anti tumoral drug emodin adsorbed on silver nanoparticles
}

\author{
Paz Sevilla*a,b, Raquel De-Llanos ${ }^{\mathrm{a}}$, Concepción Domingo ${ }^{\mathrm{a}}$, Santiago Sánchez-Cortés ${ }^{\mathrm{a}}$, José V. \\ García-Ramos $^{\mathrm{a}}$ \\ ${ }^{a}$ Instituto de Estructura de la Materia, Consejo Superior de Investigaciones Científicas, Serrano 121, \\ 28006-Madrid, Spain; \\ ${ }^{\mathrm{b}}$ Departamento de Química Física II, Facultad de Farmacia, Universidad Complutense de \\ Madrid, 28040-Madrid, Spain
}

\begin{abstract}
Metal nanostructures are known to amplify the spontaneous emission of fluorescent molecules by resonant coupling to external electromagnetic fields. We have used spectroscopy to characterize the structural properties of emodin molecules, a natural anthraquinone dye, and bovine serum albumin, the most abundant protein in plasma, in the presence of silver nanoparticles. Aggregation of emodin at $\mathrm{pH}=10$ and $\mathrm{pH}=6$ gives rise to SERS and MEF effects in silver colloid. We have obtained MEF spectra at acidic $\mathrm{pH}=2.9$ using two different silver nanostructures. We have also studied the change in the secondary structure of bovine serum albumin adsorbed on metal nanoparticles surface. Circular dichroism, fluorescence emission and fluorescence lifetime measurements indicate an increase in the alfa-helical content of the protein and a change in the environment of the tryptophan residues that bury in the interior of the biomolecule. This variation on the secondary structure could have further influence in the binding of the drug to form transport and regulatory complexes.
\end{abstract}

Keywords: Silver nanoparticles, MEF, fluorescence emission, circular dichroism, fluorescence lifetime, emodin, bovine serum albumin.

\section{INTRODUCTION}

Metal nanoparticles (NPs) exhibit a rich optical phenomenology due to the excitation of Plasmon resonances ${ }^{1}$. Besides larger scattering and absorption cross sections, such Plasmon resonances lead to high local field enhancements in a nearfield region around the particle giving rise to surface-enhanced Raman scattering (SERS) ${ }^{2}$ and metal-enhanced fluorescence (MEF) or surface-enhanced fluorescence (SEF) $)^{3,4}$. The possibility of combining localized surface plasmon (LSP) enhancement in metallic NPs has opened the way to new and powerful tools. Silver NPs have become efficient vehicles to store and deliver medicines. They are used for the controlled release of drugs, and albumin-drug complexes, where NPs act as carriers of drugs and liberate them on a selective basis, at the right speed and in the intended environment within the organism ${ }^{5}$. They can be used as "magic bullets" that go directly to cells of a particular tissue (e.g. cancer cells). Our goal is to exploit the effects of LSP in order to develop systems for the release of probes.

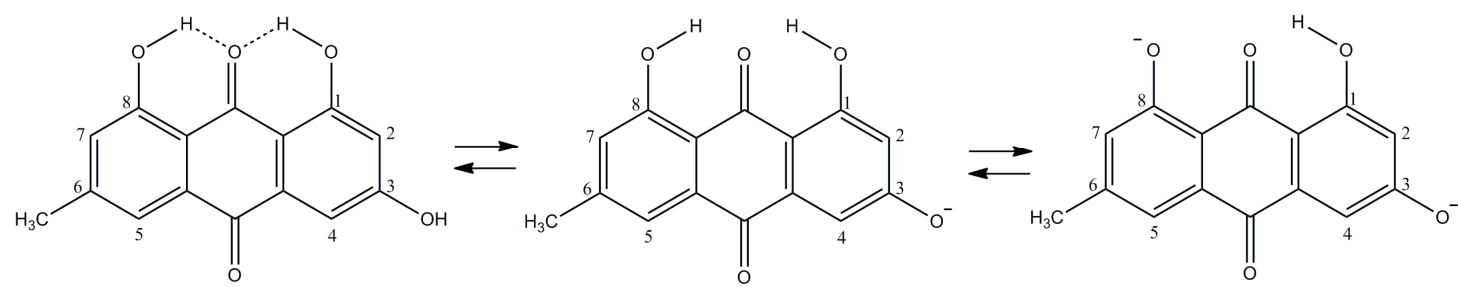

Figure 1. Structure and acid-base equilibrium of emodin

*paz@farm.ucm.es; phone 3491 3941751; fax 34913942032

Plasmonics in Biology and Medicine VII, edited by Tuan Vo-Dinh, Joseph R. Lakowicz, Proc. of SPIE Vol. 7577, $757714 \cdot$ · C 2010 SPIE · CCC code: 1605-7422/10/\$18 · doi: 10.1117/12.839704 
Emodin (6-methyl-1,3,8-trihydroxyanthraquinone) is an orange natural antraquinone that has been commonly used by its anti inflammatory and laxative effects. It has recently revealed as an effective anti tumoral drug ${ }^{6-8}$. Emodin binds albumin ${ }^{9,10}$ presents in blood and forms complexes that are able to transport the drug along the organism. It owns three hydroxyl functional groups and it is able to establish an acid-base equilibrium as it is indicated in figure 1. Di anionic form is very soluble in water; however, mono anionic and neutral species are only soluble in organic solvents. In aqueous solution emodin self-associates forming aggregates with poor fluorescence quantum yield that limit the applicability of this technique. The use of MEF to enhance the intensity of fluorescence bands overcomes these disadvantages and could afford important information on the understanding of the biological behaviour of this molecule. We have previously studied SERS and MEF of emodin on silver colloids at basic and slightly acid $\mathrm{pHs}(\mathrm{pH}=10$ and $\mathrm{pH}=6)^{11}$, but we were not able to bring down the acidity until more acidic $\mathrm{pHs}$ because of the difficulties derived from the homo association of the dye. In this work we present a simple method to obtain $\mathrm{MEF}$ of emodin at $\mathrm{pH}=2.9$. We avoid the functionalization of the metal surface with a spacer in order to place the fluorophore at a proper distance where the metal quenching of the emission leads to MEF effect. On the other hand, we have studied the effect of LSP in the formation of the albumin-emodin complexes paying attention in the changes observed on the protein structure when it is adsorbed on the metal surface.

\section{MATERIALS AND METHODS}

Emodin was purchased from Sigma-Aldrich. It was firstly solved on dimethylsulfoxide and then aliquots were added to the corresponding solution until the desired concentration. The final $\mathrm{pH}$ was adjusted with $\mathrm{NaOH}$ or $\mathrm{NO}_{3} \mathrm{H}$. Silver colloids were produced by sodium citrate ${ }^{12}(\mathrm{AgCt})$ or hydroxylamine hydrochloride ${ }^{13}(\mathrm{AgHx})$ reduction. Two types of MEF experiments have been recorded using a Confocal Raman Microscope Renishaw RM2000 provided with an exciting line at $514.5 \mathrm{~nm}$ by an $\mathrm{Ar}$ + laser, both with micro (L-50X/0.55) and macro (f:30mm) objectives. Metal nanostructures were immobilized on glass substrate by direct deposition of the colloidal suspension and then subsequent evaporation. Subsequently, emodin aqueous solution with $\mathrm{pH}=2.9$ was deposited on the silver surface and a new solvent evaporation was carried out.

Bovine serum albumin used was fatty acid free $\left(\mathrm{BSA}_{\mathrm{f}}\right)$, it was purchase from Sigma-Aldrich and used without further purification. Samples were prepared by direct addition of the solid protein to AgHx colloid. The $\mathrm{pH}$ was then adjusted to $\mathrm{pH}=6.5$. Fluorescence steady-state experiments were performed on a Perkin Elmer LB-45 and the excitation wavelength was adjusted to $\lambda_{\text {exc }}=295 \mathrm{~nm}$. Far UV-Circular dichroism (CD) measurements were performed on a JASCO-710 spectropolarimeter between 200 and $240 \mathrm{~nm}$. Time-resolved fluorescence experiments were measured on an EasyLife $\mathrm{V}^{\mathrm{TM}}$ lifetime fluorometer using the stroboscopic technique. A diode LED of $295 \mathrm{~nm}$ providing a pulsed light was used as excitation source. Fluorescence decays can be described as a sum of exponential functions:

$$
D(t)=\sum_{i=1}^{n} a_{i} \exp \left(-\frac{t}{\tau_{i}}\right)
$$

where $\tau_{i}$ are the fluorescence lifetimes of various fluorescent forms and $\mathrm{a}_{\mathrm{i}}$ are the pre-exponential factors, that in the case of multiple lifetimes resulting from the presence of several fluorophores in the sample depend not only on their populations, but also on the radiative probability constants and the molar extinction coefficients of the respective fluorophores. The amplitude-weighted lifetime is given by:

$$
\langle\tau\rangle=\sum_{\mathrm{i}=1}^{\mathrm{n}} \mathrm{a}_{\mathrm{i}} \tau_{\mathrm{i}}
$$



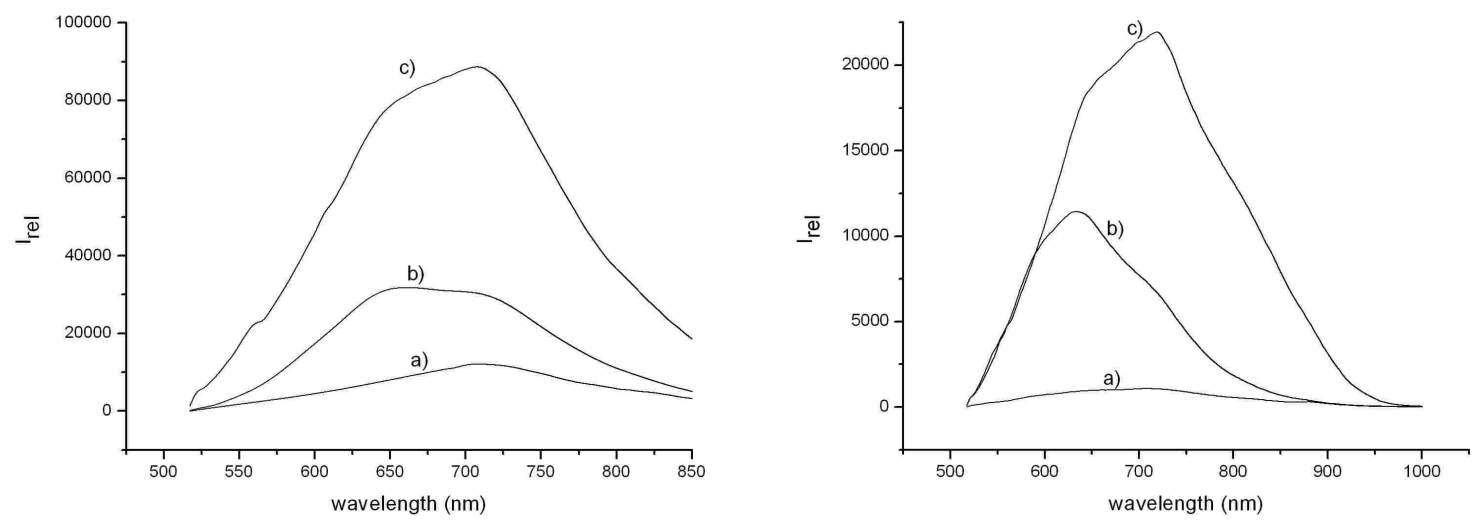

Figure 2. MEF experiments of emodin, $\lambda_{\text {exc }}=514,5 \mathrm{~nm}$. a) Over glass slide, b) over glass slide covered with Ag NPs (reduction with hydroxylamide hydrochloride, AgHx colloid), c) over glass slide covered with Ag NPs (reduction with sodium citrate, $\mathrm{AgCt}$ colloid). Left: macro (f:30mm objective). Right: micro (L-50x/N.A. 0.55 objective).

\section{RESULTS AND DISCUSSION}

MEF experiments obtained using macro and micro objectives appear in figure 2. The enhancement in fluorescence relative intensity over metal substrate is appreciable under these conditions, however, no SERS effect can be observed. This can be caused by the highly enhanced fluorescence intensity hiding the Raman emission. The absence of a molecule used as spacer indicates the presence of emodin homoassociates acting as autospacers; the same occurred at $\mathrm{pH}=6 \mathrm{when}$ silver colloid was used as metal $\mathrm{NPs}^{11}$. In the two cases, macro and micro MEF experiments, sodium citrate used as reducing agent gives rise to better enhancement than hydroxylamine hydrochloride does. On the contrary, at $\mathrm{pH}=6^{11}$ $\mathrm{AgHx}$ colloid displays better enhancement than AgCt colloid either for SERS or MEF effect.

According to the $\mathrm{UV}$-visible experiments ${ }^{11}$ at $\mathrm{pH}=2.92$ emodin is principally under the neutral specie and it is able to form $\mathrm{H}$-aggregates and/or J-aggregates by $\pi-\pi^{*}$ molecular stacking interaction. The predominance of $\mathrm{H}$ or J-aggregates depends on the colloid used to prepare the metal substrate for the MEF experiments.
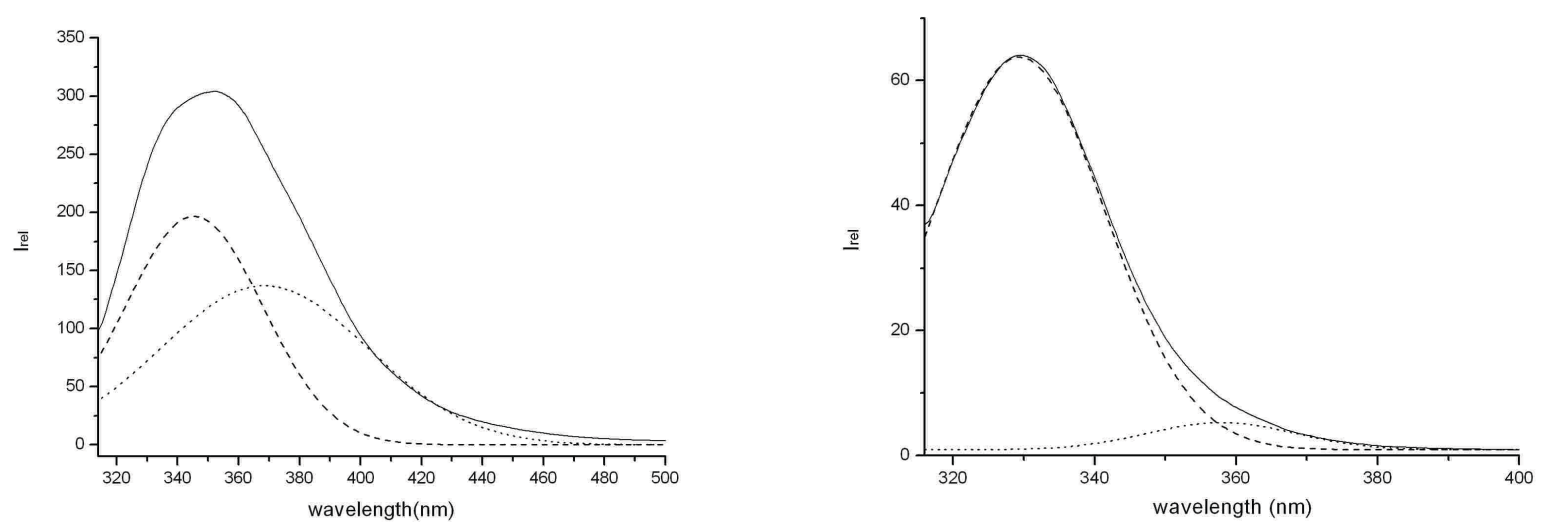

Figure 3. Fluorescence emission spectra of $\mathrm{BSA}_{\mathrm{f}}, \lambda \mathrm{exc}=295 \mathrm{~nm}$, solid line corresponds to the experimental result, dash line

(Trp-213) and dots (Trp-314) show the result for the experimental curve deconvolution Trp-213. Left: BSA $_{f}$ in aqueous solution ( $\mathrm{pH}=6.5$ ). Right: $\mathrm{BSA}_{\mathrm{f}}$ in $\mathrm{AgHx}$ colloid. 
$\mathrm{J}$-aggregates are the principal components when $\mathrm{AgCt}$ colloid is used on the contrary $\mathrm{H}$-aggregates are the most intense band when AgHx colloid does. Position of the aggregates bands in MEF experiments is red shifted respect to the bands on silver colloid ${ }^{11}$, these changes can be attributed to the adsorbate aggregation and different size of the homoassociates or on the other side by the fast-dynamics SEF (FDSEF) $)^{14,15}$.

To study the influence of the LSP on the $\mathrm{BSA}_{\mathrm{f}}$ structure when it is adsorbed on the metal surface we have recorded two types of experiments on AgHx colloid: CD and fluorescence. Mean Residue Ellipticity (MRE) exhibits a typical profile of an alfa-helical protein ${ }^{16}$ with two minimum at $208 \mathrm{~nm}$ and $222 \mathrm{~nm}$. Value for $\mathrm{MRE}_{222}$ changes from -20000 deg. $\mathrm{cm}^{2} . \mathrm{dmol}^{-1}$ in water solution to $-50000 \mathrm{deg} . \mathrm{cm}^{2} . \mathrm{dmol}^{-1}$ when protein is adsorbed on the silver NPs, thus indicating an increase in the helical contents of $\mathrm{BSA}_{\mathrm{f}}$. Fluorescence emission spectra of $\mathrm{BSA}_{\mathrm{f}}$ (figure 3 ) is due to the triptophan (Trp) residues present in the aminoacid sequence, Trp-213 buried in the protein, near the binding sites Sudlow's site I and site II, and Trp-134 exposed to the solvent and far from the binding sites ${ }^{17}$. Experiments show a broad band with a maximum at $352 \mathrm{~nm}$ when it is solved in water solution that is quenched and blue-shifted to $330 \mathrm{~nm}$ when protein is solved on AgHx colloid. The shift indicates a change in the Trp surroundings and implies lesser exposition to the solvent. Both results, $\mathrm{CD}$ and fluorescence emission, are in agreement. In order to separate participation of the two Trp on the fluorescence band we have deconvoluted the experimental curve and results are shown in figure 3. The two aminoacids exhibit similar blue-shift of the maximum. However, energy transfer between the adsorbate and the metal is not the same for every Trp. Residue Trp-213 exhibits a 67\% quenching of the relative fluorescence intensity while Trp-134 is almost negligible and the relative fluorescence intensity shows a $95 \%$ quenching.

Table 1. Lifetime decay parameters obtained for emodin in water solution and deposited on $\mathrm{Ag} \mathrm{Hx}$ colloid, $\mathrm{pH}=6.5$. $<\tau>$ is the amplitude- weighted lifetime calculated with eq. (2) $\underset{\text { to }}{\text { and }} \chi^{2}$ is the reduced chi-square statistic calculated according

\begin{tabular}{|ccc|}
\hline & $<\tau>(\mathrm{ns})$ & $\chi^{2}$ \\
$\mathrm{BSA}_{\mathrm{f}}$ in water & 3.7 & 1.11 \\
$\mathrm{BSA}_{\mathrm{f}}$ in $\mathrm{AgHx}$ & 2.8 & 1.13 \\
\hline
\end{tabular}

Finally we have calculated the fluorescence amplitude-weighted lifetime of the protein using a two exponential fitting of the decay curves. The values obtained are shown in table 1 . The observed decrease for $\langle\tau\rangle$ when protein is adsorbed on metal surface indicates Trp are more exposed to solvent in $\mathrm{BSA}_{\mathrm{f}}$ on water solution than adsorbed on silver nanostructures, due probably to the existence of more contacts with peptides bonds as a consequence of the formation of more alpha-helix in the secondary structure.

In this work we have established conditions for obtaining macro and micro MEF experiments of emodin, an anti tumoral drug, at acidic $\mathrm{pH}=2.9$. Metal substrate prepared with $\mathrm{Ag} \mathrm{Ct}$ colloid gives rise to better enhancement than the one prepared using $\mathrm{AgHx}$ colloid, unlike what occurs at $\mathrm{pH}=6$, where emodin on $\mathrm{AgHx}$ colloid exhibits bigger enhancement than emodin on Ag Ct colloid. We observe a change in the secondary structure of the $\mathrm{BSA}_{\mathrm{f}}$, when protein is adsorbed in the metal surface, becoming more helical. This is indicating by: i) a decrease in the molar residue ellipticity observed at $208 \mathrm{~nm}$, ii) a blue-shifted of the maximum of the fluorescence emission band, iii) a decrease in the amplitude-weighted lifetime. We continue working in these systems trying to deep in the changes on the structure of the protein and emodinprotein complexes adsorbed on metal NPs.

\section{ACKNOWLEDGEMENTS}

This work has been financially supported by projects: FIS2007-63065 from the Spanish Ministerio de Educación y Ciencia, S-0505/TIC/0190 from Comunidad Autónoma de Madrid and Research group 950247 from the UCM support. R. de Llanos acknowledges Ministerio de Ciencia e Innovación for a FPI fellowship. 


\section{REFERENCES}

1. Love, S.A., Marquis, B.J., Haynes, C.L., "Recent Advances in Nanomaterial Plasmonics: Fundamental Studies and Applications," Appl. Spectrosc., 62, 12, 346A-362A (2008).

2. Aroca, R., [Surface-Enhanced Vibrational Spectroscopy], John Wiley \& Sons Ltd, Chichester, England (2006).

3. Aslan, K., Gryczynski, I., Malicka, J., Matveeva, E., Lakowicz, J.R., Geddes, C.D., "Metal-enhanced fluorescence: an emerging tool in biotechnology," Curr. Opin. Biotechnol., 16, 1, 55-62 (2005).

4. Fort, E., Gresillon, S., "Surface enhanced fluorescence," J. Phys. D-Appl. Phys., 41, 1, 013001 (2008).

5. Del Valle, E.M.M., Galan, M.A., Carbonell, R.G., "Drug Delivery Technologies: The Way Forward in the New Decade,” Ind. Eng. Chem. Res., 48, 5, 2475-2486 (2009).

6. Morris, J.B., [Legume genetic resources with novel "value added" industrial and pharmaceutical use], In: J. Janick (ed.), Perspectives on new crops and new uses. ASHS Press, Alexandria, VA., 196-201 (1999).

7. Huang, Z.W., Chen, G.C., Shi, P., "Effects of emodin on the gene expression profiling of human breast carcinoma cells," Cancer Detect. Prev., 32, 4, 286-291 (2009).

8. Srinivas, G., Babykutty, S., Satbiadevan, P.P., Srinivas, P., "Molecular mechanism of emosin action: transition from laxative ingredient to an antitumor agent," Med. Res. Rev., 27, 5, 591-608 (2008).

9. Fabriciova, G., Sanchez-Cortes, S., Garcia-Ramos, J.V., Miskovsky, P., "Surface-enhanced Raman spectroscopy study of the interaction of the antitumoral drug emodin with human serum albumin," Biopolymers 74, 1-2, 125-130 (2004).

10. Sevilla, P., Rivas, J.M., Garcia-Blanco, F., Garcia-Ramos, J.V., Sanchez-Cortés, S., "Identification of the antitumoral drug emodin binding sites in bovine serum albumin by spectroscopic methods," BBA-Proteins Proteomics, 1774, 11, 1359-1369 (2007).

11. Sevilla, P., Garcia-Blanco, F., Garcia-Ramos, J.V., Sanchez-Cortes, S., "Aggregation of antitumoral drug emodin on Ag nanoparticles: SEF, SERS and fluorescence lifetime experiments," Phys. Chem. Chem. Phys., 11, 37, 8342-8348 (2009).

12. Lee, P.C., Meisel, D., “Adsorption and surface-enhanced Raman of dyes on silver and gold sols," J. Phys. Chem., 86, 17, 3391-3395, (1982).

13. Nicolae L., Bernhard L., "A New Method for Fast Preparation of Highly Surface-Enhanced Raman Scattering (SERS) Active Silver Colloids at Room Temperature by Reduction of Silver Nitrate with Hydroxylamine Hydrochloride," J. Phys. Chem. B, 107, 24, 5723-5727, (2003).

14. Ringler, M., Schwemer, A., Wunderlich, M., Nichtl, A., Kurzinger, K., Klar, T.A., Feldmann, J., "Shaping emission spectra of fluorescent molecules with single plasmonic nanoresonators," Phys. Rev. Lett., 100, 20, 203002 (2008).

15. Le Ru, E.C., Etchegoin, P.G., Grand, J., Felidj, N., Aubard, J., Levi, G., "Mechanisms of spectral profile modification in surface-enhanced fluorescence," J. Phys. Chem. C, 111, 44, 16076-16079 (2007).

16. Fasman, G.D., [Prediction of protein structure and the principles of protein conformation], Plenum Press, New York (1989).

17. Peters, T., Jr., [All about albumin: biochemistry, genetics and medical applications], Academic Press, INC., San Diego, California (1995).

18. James, D.R., Siemiarczuk, A., Ware, W.R., "Stroboscopic optical boxcar technique for the determination of lifetimes,” Rev. Sci. Instrum., 63, 2, 1710-1716 (1992). 